Provided for non-commercial research and education use. Not for reproduction, distribution or commercial use.

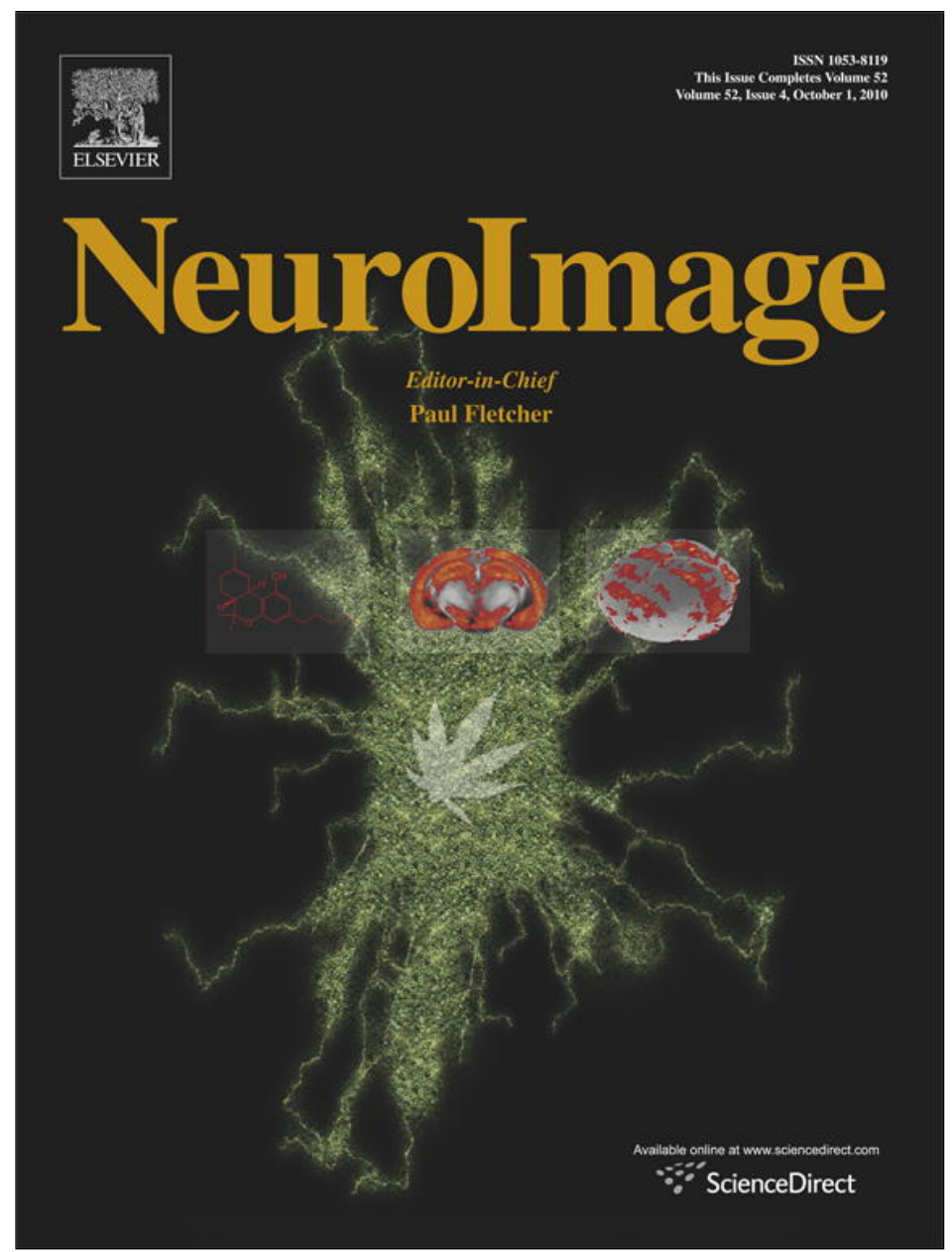

This article appeared in a journal published by Elsevier. The attached copy is furnished to the author for internal non-commercial research and education use, including for instruction at the authors institution and sharing with colleagues.

Other uses, including reproduction and distribution, or selling or licensing copies, or posting to personal, institutional or third party websites are prohibited.

In most cases authors are permitted to post their version of the article (e.g. in Word or Tex form) to their personal website or institutional repository. Authors requiring further information regarding Elsevier's archiving and manuscript policies are encouraged to visit:

http://www.elsevier.com/copyright 


\title{
Selective functional integration between anterior temporal and distinct fronto-mesolimbic regions during guilt and indignation
}

\author{
Sophie Green ${ }^{\text {a }}$, Matthew A. Lambon Ralph ${ }^{\text {a }}$, Jorge Moll ${ }^{\text {b }}$, Emmanuel A. Stamatakis ${ }^{\text {d, }}$ \\ Jordan Grafman ${ }^{\mathrm{c}}$, Roland Zahn ${ }^{\mathrm{a}, \mathrm{c}, *}$ \\ ${ }^{a}$ The University of Manchester, School of Psychological Sciences, Neuroscience and Aphasia Research Unit, Manchester, M13 9PL, UK \\ b Cognitive and Behavioral Neuroscience Unit, D'Or Institute for Research and Education (IDOR), 22280-080 Rio de Janeiro, RJ, Brazil \\ c National Institutes of Health, National Institutes of Neurological Disorders and Stroke, Cognitive Neuroscience Section, Bethesda, MD 20892-1440, USA \\ d School of Clinical Medicine, Division of Anaesthesia, University of Cambridge, Cambridge, UK
}

\section{A R T I C L E I N F O}

\section{Article history:}

Received 18 February 2010

Revised 12 May 2010

Accepted 13 May 2010

Available online 21 May 2010

\section{Keywords:}

Moral emotions

fMRI

Psychophysiological interaction

Anterior temporal lobe

Subgenual cingulate cortex

Septal region

Orbitofrontal cortex

Semantics

Guilt

Indignation

Anger

Major depression

\begin{abstract}
A B S T R A C T
It has been hypothesized that the experience of different moral sentiments such as guilt and indignation is underpinned by activation in temporal and fronto-mesolimbic regions and that functional integration between these regions is necessary for the differentiated experience of these moral sentiments. A recent fMRI study revealed that the right superior anterior temporal lobe (ATL) was activated irrespective of the context of moral feelings (guilt or indignation). This region has been associated with context-independent conceptual social knowledge which allows us to make fine-grained differentiations between qualities of social behaviours (e.g. "critical" and "faultfinding"). This knowledge is required to make emotional evaluations of social behaviour. In contrast to the context-independent activation of the ATL, there were context-dependent activations within different fronto-mesolimbic regions for guilt and indignation. However, it is unknown whether functional integration occurs between these regions and whether regional patterns of integration are distinctive for the experience of different moral sentiments. Here, we used fMRI and psychophysiological interaction analysis, an established measure of functional integration to investigate this issue. We found selective functional integration between the right superior ATL and a subgenual cingulate region during the experience of guilt and between the right superior ATL and the lateral orbitofrontal cortex for indignation. Our data provide the first evidence for functional integration of conceptual social knowledge representations in the right superior ATL with representations of different feeling contexts in fronto-mesolimbic regions. We speculate that this functional architecture allows for the conceptually differentiated experience of moral sentiments in healthy individuals.
\end{abstract}

(c) 2010 Elsevier Inc. All rights reserved.

\section{Introduction}

The ability to be motivated by moral values is uniquely human (Fehr and Fischbacher, 2003). The 18th century British philosophers, Adam Smith and David Hume suggested that our behaviour is guided by intuitive "moral sentiments" which express our moral approval or disapproval (Lamb, 1974). Here, we focus on moral sentiments related to violation of values that are of particular relevance for the understanding of common psychiatric disorders such as major depression (Berrios et al., 1992; O'Connor et al., 2002). We may experience different types of moral sentiments depending on who is the agent of a moral violation. Self-blaming sentiments, such as guilt are associated with self-agency, whereas other-blaming sentiments such as indig-

\footnotetext{
* Corresponding author. The University of Manchester, School of Psychological Sciences, Neuroscience and Aphasia Research Unit, Manchester, M13 9PL, UK. Fax: +44 1612752873.

E-mail address: roland.zahn@manchester.ac.uk (R. Zahn).
}

nation are associated with another person being the social agent (Zahn et al., 2009a).

Previous fMRI studies have revealed activation of the anterior temporal lobe (ATL) and fronto-mesolimbic regions in response to morally relevant stimuli that often evoke the experience of moral sentiments (Heekeren et al., 2005; Moll et al., 2002a, 2001; Shin et al., 2000; Takahashi et al., 2004). It has been hypothesized that the experience of such moral sentiments (e.g. guilt and indignation) is underpinned by integration between ATL and fronto-mesolimbic regions (Moll et al., 2005; Zahn et al., 2009a). In a previous study using fMRI, we found activation of the right superior anterior temporal lobe (ATL) that was present irrespective of the type of the moral sentiment experienced (i.e. context-independent). This stable ATL activation was accompanied by context-dependent activations within the subgenual cingulate cortex (extending into the septal area) selective for guilt and within the lateral orbitofrontal cortex (OFC) for indignation.

The context-independent activation of the right superior ATL during the experience of both guilt and indignation is consistent with 
a model which considers the ATL as a hub that represents abstract and concrete conceptual knowledge that is context-independent and necessary for differentiation and generalization of meaning (Lambon Ralph and Patterson, 2008; Lambon Ralph et al., 2009; Lambon Ralph et al., 2010; Patterson et al., 2007; Rogers et al., 2006, 2004). According to this model, the ATL allows for the generation of "coherent concepts" across different domains and modalities (Lamambon Ralph et al., 2010) such that objects and actions can be identified, understood and generalized across different contexts. ATL activation during fMRI has also been found when probing personrelated information (Simmons et al., 2009; Tsukiura et al., 2008; Campanella and Belin, 2007). Further, the inferior anterior temporal region has been demonstrated to allow identification of faces (Kriegeskorte et al., 2007). Although, Simmons et al. (2009) were able to detect ATL activations for person-related information, they did not find activations for non-social inanimate objects as would have been predicted by a domain-general account of ATL function. This may be explained by the use of tasks that probe newly learned associations between object names (e.g. "The Newport building") and functional features ("is used for voter registration") that are not part of the generalizable definition of the coherent concept.

Recent evidence implicates the superior aspect of the right ATL in the representation of context-independent conceptual social knowledge which allows us to differentiate between different social behaviours (e.g. 'stingy,' 'generous,' 'honest' or 'brave') (Zahn et al., 2007, 2009a,b), and is necessary to understand and emotionally evaluate the behaviours of ourselves and others. We hypothesized that these context-independent, conceptual representations of social behaviour can be flexibly embedded within different feeling contexts (Moll et al., 2005; Zahn et al., 2009a). This conceptual social knowledge was hypothesized to be an integral part of the experience of moral sentiments, acting to enrich feelings with differentiated meaning (Moll et al., 2005).

In addition to this context-independent ATL activation there was context-dependent activation of the subgenual cingulate cortex extending into the septal area during the experience of guilt (Zahn et al., 2009a). The septal region comprises the ventral part of the human septum (i.e. "septum verum") and can be distinguished from the septum pellucidum (Andy and Stephan, 1968). The septum verum contains a group of basal forebrain nuclei that have greatly increased in size with phylogeny from lower animals to humans (Andy and Stephan, 1968). Previous fMRI studies have found activations in various parts of the septal region and the adjacent subgenual cingulate cortex (further referred to as septal region/subgenual cingulate cortex (SSC)) for tasks that are associated with affiliative feelings such as altruistic (Moll et al., 2006), and trust-based decisions (Krueger et al., 2007), as well as for both passive viewing (Zahn et al., 2009c) and emotional judgements of guilt-evoking stimuli (Zahn et al., 2009a). These studies did not specifically dissociate the emotional and cognitive aspects of affiliative feelings as they are highly interdependent (Moll et al., 2005). Guilt is ultimately pro-social in nature (Moll et al., 2008) since it is derived from our concern for the wellbeing of other individuals (Weiss et al., 1995) and enhances relationships by promoting maintenance of attachment to loved ones (Baumeister et al., 1994). Thus, the activation of the SSC during the experience of guilt is in line with evidence suggesting that this region is involved in affiliative feeling contexts. We speculate that parts of the SSC region may represent affiliative values of social behaviours which are important for evaluating, planning and choosing between different social actions.

Interestingly, abnormalities in the subgenual cingulate cortex have been found consistently in patients with major depression (Drevets and Savitz, 2008; Ebert and Ebmeier, 1996; Ressler and Mayberg, 2007). These patients often experience guilt in an "inappropriate" manner (DSM-IV, American Psychiatric Association, 2000); that is, the experience of guilt becomes overgeneralized to inappropriate contexts. Patients may also feel something which they clearly label as guilt, but are unable to tie to a specific transgression or misdeed (Berrios et al., 1992). It has been hypothesized that in order to experience guilt in a differentiated fashion, conceptual social knowledge in the ATL must be integrated with affiliative feeling contexts represented in the SSC (Moll et al., 2008; Zahn et al., 2009c). If such conceptual knowledge is not well integrated, then guilt may be experienced inappropriately as it is in major depression (Berrios et al., 1992; O'Connor et al., 2002).

In contrast to the guilt-selective activation of the SSC, the experience of indignation was associated with activation of the lateral OFC (Zahn et al., 2009a), one of a number of regions including the insula and dorsomedial prefrontal cortex (PFC) that have been implicated in the experience of negative emotions such as anger, disgust and contempt (Blair et al., 1999; Calder et al., 2000; Fitzgerald et al., 2004; Seymour et al., 2007). That this region is activated during the experience of negative moral sentiments is in accordance with its role in the representation of non-affiliative punishment/reward values and outcomes of both sensory, (Rolls, 2004; Small et al., 2001) and abstract reinforcers such as money (O'Doherty et al., 2001).

Although the above results suggest that the SSC, lateral OFC and ATL play an important role in the experience of guilt and indignation, it is unknown whether functional integration occurs between these regions and whether there are distinct patterns of integration for different moral sentiments. It is well established that cognitive functions depend on functional integration across anatomically distributed networks (Friston, 2010), yet conventional fMRI analyses are not suited to investigate which regions within a given network interact to support a given cognitive function. Standard analyses of Blood-Oxygenation-Level-Dependent (BOLD) effects revealing two brain regions responding to a psychological stimulus do not indicate that there are psychologically and physiologically relevant interactions occurring between these areas. Psychophysiological interaction (PPI) analyses, however, can elucidate whether functional integration occurs between regions and whether this integration changes during different psychological contexts (Friston et al., 1997). PPI analysis is based on a regression model which includes the interaction term of the psychological variable convolved with an estimate of the neural activity in a seed region, as a predictor variable. As such, this analysis probes the association between two brain regions during a given psychological condition. Thus, a change in the association between the two brain regions due to a change in the psychological context would be reflected in the change of the regression slope. If this change is significant, a significant PPI effect is said to occur (Friston et al., 1997). Thus, PPI analyses reveal fundamentally different information to standard BOLD effect analyses that do not explore interactions or coupling of activation between regions.

Here, we used PPI analysis with a right superior ATL seed region to test two hypotheses: 1) There is selective integration between the right superior ATL and SSC during the experience of guilt compared with indignation, and 2) There is selective integration between the right superior ATL and lateral OFC for indignation compared with guilt.

\section{Materials and methods}

Here, we briefly summarize the fMRI methods we used in this paper. Standard BOLD effect results, imaging and behavioural data acquisition methods have previously been reported (Zahn et al., 2009a).

\section{Participants}

Data from 25 healthy participants (12 men, 13 women, age: mean $=27.6 \pm 7.7$ years, education: mean $=17.2 \pm 1.6$ years) of the 29 that took part in a previous fMRI experiment (see Zahn et al. (2009a) for full methods) were included. Four participants were 
excluded because of an insufficient number of trials rated as guiltevoking for the purpose of PPI analysis. All were right handed, native English speakers and had no history of systemic, psychiatric or neurological disorders, or psychopharmacological treatment. All had passed a neurological examination and a clinical screening MRI during the previous 12 months and had normal or corrected-to-normal vision. Participants had not taken any centrally active medications or consumed alcohol $24 \mathrm{~h}$ prior to scanning. Informed consent and compensation were in accordance with NINDS standards and were approved by the NINDS internal review board.

\section{fMRI paradigm}

Participants were presented with written statements using social concepts (e.g., 'stingy,' 'tactless') describing the participants' social behaviour towards their best friend ("self-agency" condition, 50\% of trials) or their best friend's social behaviour towards them ("otheragency" condition, $50 \%$ of trials). These social behaviours were either in accordance with (positive-valence condition) or counter to (negative-valence condition) social values. For example in the positive self-agency condition: "[participant's name] acts generously towards [best friend's name]," positive other-agency condition: "[best friend's name] acts generously towards [participant's name]," negative self-agency condition: "[participant's name] acts stingily towards [best friend's name], and negative other-agency condition: "[best friend's name] acts stingily towards [participant's name]." Thus, there were five conditions: 1$)$ positive self-agency $(n=45) ; 2)$ negative self-agency $(n=45) ; 3)$ positive other-agency $(n=45) ; 4)$ negative other-agency $(n=45)$; and 5) fixation of visual pattern (null event, $n=90)$. Stimuli were presented in an event-related design, for $4 \mathrm{~s}$, followed by a jittered inter-trial interval with a mean duration of $4.6 \mathrm{~s}$, in a pseudo-random order across three runs. During the scan, participants had to decide whether the described situations would feel pleasant or unpleasant After the scan participants rated each statement on pleasantness/ unpleasantness (1- to 7-point Likert scale) and chose the label that best described their feeling (from their own perspective) from a choice of: pride, gratitude, guilt, embarrassment, indignation/anger, none/other. On the first level individual subject analysis, we only included trials that the individual rated as guilt-evoking during the negative self-agency condition and trials rated as indignation/anger-evoking during the negative other-agency condition.

\section{Image acquisition}

Echo-planar T2*-weighted images were acquired (344 volumes per run) on a 3 Tesla General Electric scanner equipped with a standard head coil, high-order manual shimming to temporal and ventral frontal lobes, $3 \mathrm{~mm}$ slice thickness, $64 \times 64$ matrix, 37 slices, $\mathrm{TR}=2.3 \mathrm{~s}, \mathrm{TE}=20.5$, FOV: $220 \times 220 \mathrm{~mm}$, parallel to the anterior to posterior commissural line, whole-brain coverage (not cerebellum). The first five volumes were discarded. The combination of high-field MRI, thinner slices (Bodurka et al., 2007) and high-order manual shimming optimized the signal in anterior temporal and ventral frontal lobes. All participants had full coverage of the ATL and most of the ventral frontal cortex upon inspection of normalized echoplanar images. In addition, high resolution $\left(\approx 1 \mathrm{~mm}^{3}\right)$ T1-weighted 3D Magnetization-Prepared Rapid Acquisition Gradient Echo structural images were collected ( $1 \mathrm{~mm}$ slice thickness, 128 slices, matrix: $224 \times 224, \mathrm{TE}=2.964 ;$ FOV: $220 \times 222 \mathrm{~mm}$ )

\section{Imaging analysis}

\section{Pre-processing}

Functional images were realigned, unwarped and slice time corrected. These images were normalized by first normalizing the participants' T1 image ( $3 \mathrm{~mm}^{3}$ voxel size) to the Statistical Parametric
Mapping (SPM5) T1-template and then applying the same transformation to the functional images. A smoothing kernel of FWHM $=6 \mathrm{~mm}$ was used. Psychophysiological Interaction (PPI) Analysis was performed using Statistical Parametric Mapping 5 (SPM5, http://www.fil.ion.ucl. ac.uk/spm/software/spm5).

\section{Psychophysiological interaction analysis}

PPI requires the extraction of the signal of the seed region (in this case, the right superior ATL) and the interaction term which is the multiplication of the psychological variable with the physiological variable. A whole-brain search identifies all voxels whose variance in activation can be explained by the psychophysiological interaction term.

The seed region was a sphere with a radius of $6 \mathrm{~mm}$ constructed around the peak coordinate of the ATL activation in Zahn et al. (2009a); ( $x=57, y=-3, z=-6$; all coordinates throughout the text are in Montreal Neurological Institute (MNI) space). The neural time series of the seed region was estimated by deconvolving the BOLD response using the standard deconvolution algorithm in SPM5 (Gitelman et al., 2003).

At the single subject level, the physiological variable was extracted and the psychophysiological interaction terms were created for guilt vs. null, indignation vs. null and entered into a common general linear model. The following contrast images were computed at the individual subject level: guilt vs. null, indignation vs. null, indignation vs. guilt, guilt vs. indignation. After performing PPI group level randomeffects analyses of guilt vs. null and indignation vs. null with an uncorrected threshold of $p=0.05$, the statistical parametric maps were saved and used for inclusive masking of the guilt vs. indignation and indignation vs. guilt contrasts, respectively. Previously it has been shown that activation associated with the experience of guilt is affected by the frequency with which individuals experience guilt during the experiment (Zahn et al., 2009a). Thus, the frequency with which individuals experienced guilt and indignation during the experiment were transformed into z-scores (participants' scores were transformed based on a normative sample of $n=64$ healthy individuals, to remove differences in the mean frequency between moral sentiments (Zahn et al., 2009a)) and both were entered into the guilt vs. indignation and indignation vs. guilt random-effects PPI models as covariates. For guilt vs. null, individual frequencies of guilt were entered as covariates of interest and for indignation vs. null, individual frequencies of indignation were entered as a covariate of interest. For guilt vs. indignation, guilt was entered as a covariate of interest and indignation as a covariate of no interest. For indignation vs. guilt, frequency of indignation was entered as a covariate of interest and frequency of guilt as a covariate of no interest.

There was a significant positive correlation between individual frequencies of guilt and indignation $(r=0.53, n=25, p=0.007,2$ tailed), however, there was no relevant multicollinearity between these covariates (variance inflation factor $=1.35$ ).

Here we report the following contrasts: guilt vs. indignation inclusively masked with guilt vs. null, and indignation vs. guilt, inclusively masked with indignation vs. null. We had two reasons for looking at complex contrasts (e.g. guilt vs. indignation) and inclusively masking those contrasts with simple contrasts versus the null condition (e.g. guilt vs. null): Firstly, simple contrasts of guilt vs. null and indignation vs. mull do not establish whether seemingly specific regions for one sentiment would survive direct statistical comparison against the other sentiment. Secondly, comparing the PPI effects for moral sentiments to the null condition only (e.g. guilt vs. null) does not reveal whether effects are due to increased coupling during the moral sentiment of interest, or decreased coupling during the null condition. By comparing guilt vs. indignation and inclusively masking with guilt vs. mull, we were able to ensure that effects seen were due to positive coupling of the ATL with other brain areas for guilt, rather than a decrease in coupling for indignation, since only effects in those 
voxels that also showed positive coupling for guilt vs. null survive the masking.

All PPI effects surviving an uncorrected voxel-level threshold of $p=0.01$ and a Family-Wise-Error (FWE)-corrected $p=0.05$ over either the whole brain or our a priori regions of interest are reported in the text and tables. After conducting the PPI analysis and examining whole-brain results at $p=0.01$, two different tiers of ROIs were used to see whether the resulting clusters survived FWE-correction for multiple comparisons. The first tier was comprised of regions that have been associated with moral sentiments in the literature (Moll et al., 2005) but that we had no specific hypotheses about. They were included given the ability of PPI to detect effects in regions previously undiscovered using conventional analyses and to ensure the specificity of our positive findings. These included: posterior superior temporal sulcus/temporo-parietal junction, anterior temporal lobes, ventromedial PFC, dorsolateral PFC, dorsomedial PFC, insula, amygdala, basal ganglia, hypothalamus and ventral tegmental area. The Automatic Anatomical Labelling atlas (AAL, (Tzourio-Mazoyer et al., 2002) implemented in the Wake Forrest University (WFU) Pickatlas tool (Maldjian et al., 2003) was used to create these ROIs (for details please see Zahn et al. (2009a) supplementary materials: (http:// cercor.oxfordjournals.org/cgi/content/full/bhn080/DC1). A frontopolar cortex (BA 10) region was created using the WFU pickatlas tool.

The second tier was comprised of regions that we had specific hypotheses about; a specific SSC and lateral OFC region. These ROIs were spheres with a radius of $5 \mathrm{~mm}$ constructed around a priori mean coordinates from previous studies and their contralateral homologues. The contralateral homologues were included because the seed coordinate for the PPI analysis was in the right hemisphere and we suspected that results may be strongest in regions ipsilateral to the seed region. The centre of the sphere for the SSC ROI $(x=-4, y=23$, $z=-5$ ) was created by averaging the peak coordinates of four separate studies that have implicated this area in the experience of guilt and other pro-social sentiments (Krueger et al., 2007; Moll et al., 2006; Zahn et al., 2009a,c). The centre coordinate $(x=41, y=33, z=-2)$ of the lateral OFC ROI was created by averaging the peak coordinates of three independent studies that linked lateral OFC activation with moral indignation/anger (Moll et al., 2007, 2006; Zahn et al., 2009b).

For graphical display, an uncorrected voxel-level threshold of $p=0.05$ with an extent threshold of 10 voxels was used for overlays on the T1 template in MRICron (http://www.sph.sc.edu/comd/rorden/ MRicron/). We used anatomical landmarks and the Talairach atlas to determine Brodmann areas in our tables (Talairach and Tournoux, 1988).

\section{Results}

\section{Imaging results}

There were no significant PPI effects for comparisons of guilt vs. indignation and indignation vs. guilt when individual frequencies of guilt and indignation were used as covariates of no interest. All results reported below are from analyses including individual frequencies of guilt and indignation as covariates of interest. There were no significant effects in any of our Tier 1 a priori ROIs (posterior superior temporal sulcus/temporo-parietal junction, anterior temporal lobes, ventromedial PFC, dorsolateral PFC, dorsomedial PFC, insula, amyg- dala, basal ganglia, hypothalamus and ventral tegmental area, frontopolar cortex). All significant effects emerged in our Tier 2 regions, the lateral OFC and SSC.

Using the ATL seed region, there was a significant PPI effect in bilateral SSC (right hemispheric peak) during the experience of guilt compared with indignation, whereas for indignation compared with guilt, there was a significant PPI effect in the right lateral OFC (see Table 1, Fig. 1). No other effects were seen in any other tier 2 region.

\section{Discussion}

A previous study has demonstrated that the right superior ATL is activated irrespective of the type of moral sentiment experienced, together with sentiment-selective activations in different frontomesolimbic regions (Zahn et al., 2009a). However, it was unknown whether there is functional integration between these regions. Using a PPI analysis, we were able to confirm our hypotheses that there is selective functional integration between the right superior ATL and the SSC for guilt and between the right superior ATL and right lateral OFC for indignation. Interestingly, the degree of functional integration between ATL and SSC during the experience of guilt increased with increasing individual frequency of guilt during the experiment. Similarly, functional integration between ATL and lateral OFC regions during the experience of indignation increased with increasing individual frequency of indignation. The selective coupling of the ATL with these two regions for guilt and indignation could not be explained by general differences in the degree of unpleasantness as this was rated as equally strong by participants (Zahn et al., 2009a).

In order to experience moral sentiments, be it guilt towards self, or indignation towards others, we must be able to understand a given social behaviour which requires us to differentiate between behaviours of different conceptual qualities (e.g. "careless," "absentminded"). By doing so, we can understand whether a social transgression has occurred and the precise nature of this transgression. It has previously been demonstrated that this conceptual social knowledge is selectively represented in the superior ATL when compared with non-social conceptual knowledge (Zahn et al., 2007) and that its activation increases with the degree of conceptual differentiation required (Zahn et al., 2007, 2009a). Selective activations for social conceptual knowledge have also been demonstrated within more middle anterior temporal lobe regions close to the superior temporal sulcus (Ross and Olson, 2010).

This is in line with evidence for the ATL being necessary for performance of implicit and explicit semantic tasks that require rapid and differentiated conceptual understanding of verbal, pictorial, olfactory and auditory stimuli (Bozeat et al., 2000b, 2003; Lambon Ralph and Patterson, 2008; Patterson et al., 2007). The ATL has also been shown to underpin generalization across and differentiation between concepts and under- and overgeneralization errors in patients with neurodegeneration of this area (Lambon Ralph and Patterson, 2008; Lambon Ralph et al., 2010). The notion that contextindependent conceptual social knowledge is represented in the right superior ATL and that it is part of the representation of moral sentiments is consistent with a broader approach to understanding the contribution of ATL regions to semantic cognition, more generally; that context-independent, semantic representations are computed in

Table 1

Regional PPI effects with right superior ATL seed region.

\begin{tabular}{|c|c|c|c|c|c|c|}
\hline \multirow[t]{2}{*}{ Comparison } & \multirow[t]{2}{*}{ Anatomical region } & \multirow[t]{2}{*}{ BA } & \multicolumn{3}{|c|}{ MNI coordinates } & \multirow[t]{2}{*}{$T$-value } \\
\hline & & & $x$ & $y$ & $z$ & \\
\hline Guilt vs. indignation ${ }^{1}$ & Bilateral subgenual cingulate cortex (right hemispheric peak) & 24 & 3 & 24 & 3 & $3.0^{*}$ \\
\hline Indignation vs. guilt ${ }^{2}$ & Right lateral orbitofrontal cortex & 47 & 45 & 30 & -3 & $3.3^{*}$ \\
\hline
\end{tabular}

Only regions surviving comparisons inclusively masked with $1=$ guilt vs. null or $2=$ indignation vs. null are reported. ${ }^{*}=$ regions surviving FWE-corrected $p=0.05$ over a priori ROIs. No other regions survived a voxel-level threshold of $p=0.01$ uncorrected and FWE-corrected $p=0.05$ over the whole brain or our a priori ROIs. 


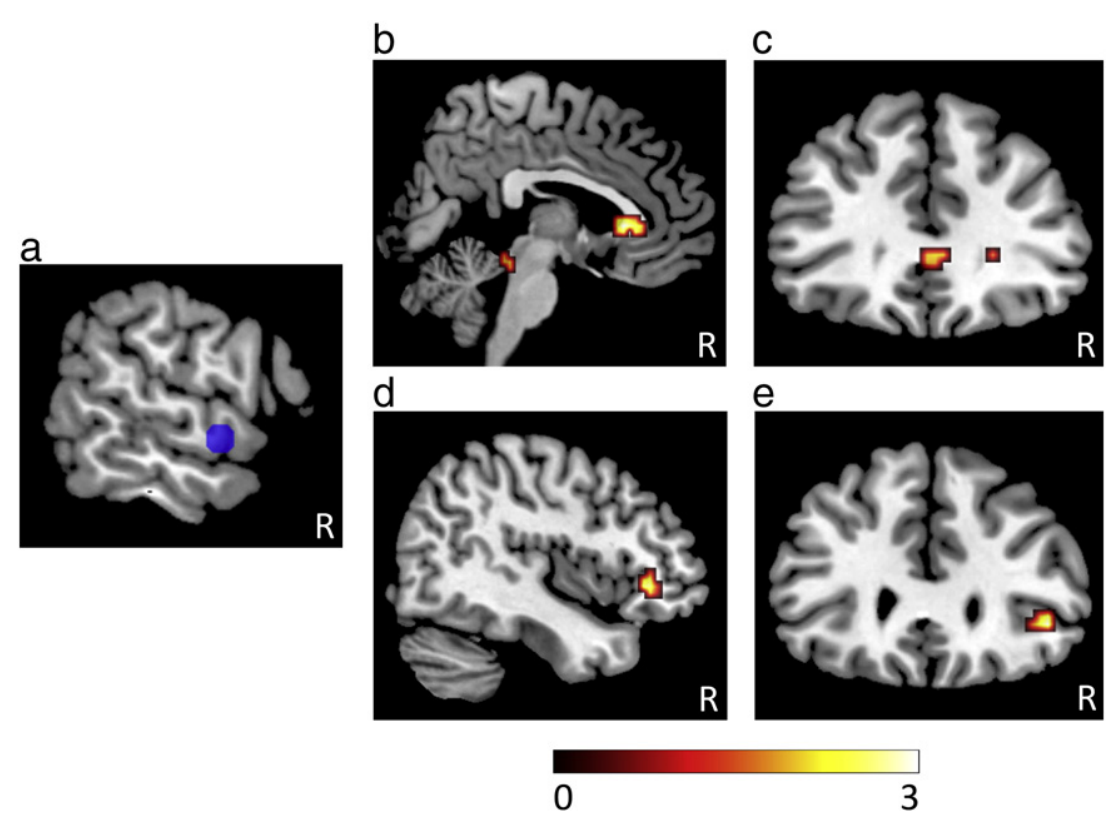

Fig. 1. Panel a) depicts the PPI seed region which consisted of a $6 \mathrm{~mm}$ sphere in the right superior ATL. Panels $\mathrm{b})$ and $\mathrm{c})$ show sagittal $(x=3)$ and coronal $(y=33)$ displays of the SSC region that exhibited PPI coupling effects with the right superior ATL seed region during guilt vs. indignation. Panels $\mathrm{d})$ and $\mathrm{e})$ show sagittal $(x=43)$ and coronal $(y=30)$ displays of the right lateral OFC region showing PPI coupling effects with the right superior ATL seed region during indignation vs. guilt using the lateral OFC ROI as a mask for display. All results were inclusively masked with comparisons vs. the null condition to show areas with positive coupling effects during the moral sentiment of interest. All areas survived FWEcorrection at $p=0.05$ over a priori ROIs and were depicted at uncorrected $p=0.05$ with an extent threshold of 10 voxels, for display purposes.

the ATL and that to generate context-specific responses, interactions with other areas are required (Jefferies and Ralph, 2006; Lambon Ralph and Patterson, 2008).

Interestingly, atrophy in the anterior temporal lobes in patients with different forms of frontotemporal lobar degeneration has been associated with a loss of moral sentiments such as pity and inappropriate social behaviour (Bozeat et al., 2000a; Edwards-Lee et al., 1997; Liu et al., 2004; Neary et al., 1998; Rankin et al., 2006). In addition, a recent study demonstrated that neurodegeneration within the right ATL in these patients led to selective impairments of contextindependent social conceptual knowledge, which was associated with inappropriate social behaviour and emotional withdrawal (Zahn et al., 2009b). These abnormalities in moral sentiments and social behaviour may result partly from loss of conceptual social knowledge representations due to atrophy of the ATL.

To our knowledge, there is only one previous fMRI study that assessed functional integration during the experience of moral feelings (Burnett and Blakemore, 2009). This study compared moral sentiments with basic emotions (e.g., fear and disgust) and demonstrated coupling of the left rather than the right superior ATL with an anterior medial PFC seed region (Burnett and Blakemore, 2009). Interestingly, in the current study, there was coupling of the right superior ATL seed region with the left superior ATL pointing towards the recruitment of a bilateral conceptual system (Lambon Ralph et al., 2009; Patterson et al., 2007), however this left ATL effect did not survive FWE-correction. More research is needed to look at factors affecting laterality of ATL activations during the experience of moral sentiments.

The coupling of the SSC with the right superior ATL during the experience of guilt is in agreement with the proposed role of the SSC in affiliative feelings (Bartels and Zeki, 2004; Moll et al., 2008, 2006; Moll and Schulkin, 2009; Zahn et al., 2009c). This coupling effect was observed in a sector of Brodmann area (BA) 24. Vogt et al. (Vogt et al., 1995,1987 ) subdivided this region into three subregions; BA 24a, 24b, $24 \mathrm{c}$ in both monkeys and humans. Our SSC cluster partly overlaps with rostro-ventral BA 24a/33 and lies partially within the callosal sulcus. Using fMRI, the SSC has been associated with a number of prosocial behaviours such as charitable donation (Moll et al., 2006), trust in economic interactions (Krueger et al., 2007) and empathic concern while experiencing guilt (Zahn et al., 2009c). Other fMRI studies of guilt have not reported activation within SSC regions (Kedia et al., 2008; Moll et al., 2007; Shin et al., 2000; Takahashi et al., 2004), however these studies did not model individual differences in the tendency to feel guilt. It has since been demonstrated that SSC activity can be detected when individual differences in the frequency of guilt experience are modelled (Zahn et al., 2009a,c). Furthermore, the current study demonstrates that integration between the SSC and other regions, namely the ATL, is affected by individual differences in proneness to experimentally induced feelings of guilt.

The SSC cluster appears to fall onto the corpus callosum when using a standard MNI template. This raises the question of whether the PPI effect could have arisen by callosal white matter rather than subcallosal grey matter signal. However, there is strong physiological evidence demonstrating that BOLD effects arise from grey matter rather than white matter (Logothetis and Wandell, 2004). Nevertheless, we conducted a supplementary analysis (see supplementary information) to rule out the possibility of the SSC cluster having purely arisen from white matter signal. We created a grey matter segmented template from our participants' structural MRIs and were able to show that at least part of the SSC cluster falls onto subcallosal grey matter. Thus, it is reasonable to attribute our PPI effect to subcallosal gyrus activity.

The PPI effects within the lateral OFC during indignation are in accordance with previous research indicating a role for the lateral OFC in other-critical moral feelings (Borg et al., 2008; Moll et al., 2007, 2005) and its role in the representation of non-affiliative reward/ punishment value (O'Doherty et al., 2001; Rolls, 2004; Small et al., 2001).

The anatomical connectivity of these regions is of particular interest when considering these functional integration effects. Tracer experiments in macaque monkeys demonstrated that the superior ATLs are directly connected with the lateral OFC (BA 47/12) (Kondo et al., 2003), and diffusion spectrum imaging and autoradiography link the superior ATL with the lateral OFC and the subgenual cingulate region via the uncinate fasciculus (Schmahmann et al., 2007). In vivo interactive dissection has also revealed connectivity between the 
temporal lobes and the ventromedial PFC in human brains (Catani et al., 2002). Our data demonstrate that despite the presence of direct anatomical superior ATL-SSC and superior ATL-OFC connections the functional contribution of the right superior ATL to activation of these areas is dynamic, changing depending on the type of moral sentiment experienced. It is highly likely that functional coupling of these regions was mediated by the known direct anatomical connections, but it is a matter of debate whether current methods of functional and effective connectivity are able to rule out mediation through indirect connections via a third area (Friston et al., 1997; Rogers et al., 2007; Stephan, 2004).

A limitation of this study was that we restricted our PPI analyses to moral sentiments related to blame rather than praise and we therefore have no direct evidence for coupling of the right superior ATL with other mesolimbic brain regions while experiencing positive moral feelings. A further limitation was that our statistical power to detect effects in other regions implicated in moral cognition more generally (Tier 1 ROIs) was lower than for our specifically predicted regions (Tier 2 ROIs), because of differences in the number of voxels over which corrections for multiple comparisons were carried out.

In conclusion, this study provides the first evidence for dynamic functional integration between the anterior temporal cortex and distinct fronto-mesolimbic regions during the experience of different moral sentiments (Moll et al., 2005). This integration may allow for the differentiated, adaptive experience of moral sentiments in healthy participants. These findings shed new light on the functional anatomy of guilt and indignation. This will be of particular relevance for future studies of individuals with major depression who are known to experience guilt in an overgeneral and maladaptive fashion (Berrios et al., 1992; O'Connor et al., 2002).

\section{Acknowledgments}

This study was supported by NINDS (USA) intramural funding to J.G. and a German National Academy of Sciences Leopoldina Fellowship funded by the Federal Ministry of Education and Research (BMBF-LPD 9901/8-122) to R.Z. S.G. was funded by an MRC PhD studentship. R.Z. was supported by a Stepping Stones Fellowship from the Faculty of Medical and Human Sciences, The University of Manchester, UK. J.M. was supported by the LABS-D'Or Hospital Network, Rio de Janeiro, Brazil. E.A.S. was funded by a Stephen Erskine Fellowship, Queens' College, Cambridge, UK.

\section{Appendix A. Supplementary data}

Supplementary data associated with this article can be found, in the online version, at doi:10.1016/j.neuroimage.2010.05.038.

\section{References}

Andy, O.J., Stephan, H., 1968. Septum in human brain. J. Comp. Neurol. 133, 383-410. Bartels, A., Zeki, S., 2004. The neural correlates of maternal and romantic love. Neuroimage 21, 1155-1166.

Baumeister, R.F., Stillwell, A.M., Heatherton, T.F., 1994. Guilt: an interpersonal approach. Psychol. Bull. 115, 243-267.

Berrios, G.E., Bulbena, A., Bakshi, N., Dening, T.R., Jenaway, A., Markar, H., Martinsantos, R., Mitchell, S.L., 1992. Feelings of guilt in major depression-conceptual and psychometric aspects. Br. J. Psychiatry 160, 781-787.

Blair, R.J.R., Morris, J.S., Frith, C.D., Perrett, D.I., Dolan, R.J., 1999. Dissociable neural responses to facial expressions of sadness and anger. Brain 122, 883-893.

Bodurka, J., Ye, F., Petridou, N., Murphy, K., Bandettini, P.A., 2007. Mapping the MRI voxel volume in which thermal noise matches physiological noise-Implications for fMRI. Neuroimage 34, 542-549.

Borg, J.S., Lieberman, D., Kiehl, K.A., 2008. Infection, incest, and iniquity: investigating the neural correlates of disgust and morality. J. Cogn. Neurosci. 20, 1529-1546.

Bozeat, S., Gregory, C.A., Lambon Ralph, M.A., Hodges, J.R., 2000a. Which neuropsychiatric and behavioural features distinguish frontal and temporal variants of frontotemporal dementia from Alzheimer's disease? J. Neurol. Neurosurg. Psychiatry 69, 178-186.
Bozeat, S., Lambon Ralph, M.A., Patterson, K., Garrard, P., Hodges, J.R., 2000b. Nonverbal semantic impairment in semantic dementia. Neuropsychologia 38 , 1207-1215.

Bozeat, S., Ralph, M.A.L., Graham, K.S., Patterson, K., Wilkin, H., Rowland, J., Rogers, T.T., Hodges, J.R., 2003. A duck with four legs: investigating the structure of conceptual knowledge using picture drawing in semantic dementia. Cogn. Neuropsychol. 20, 27-47.

Burnett, S., Blakemore, S.J., 2009. Functional connectivity during a social emotion task in adolescents and in adults. Eur. J. Neurosci. 29, 1294-1301.

Calder, A.J., Keane, J., Manes, F., Antoun, N., Young, A.W., 2000. Impaired recognition and experience of disgust following brain injury. Nat. Neurosci. 3, 1077-1078.

Campanella, S., Belin, P., 2007. Integrating face and voice in person perception. Trends Cogn. Sci. 11, 535-543.

Catani, M., Howard, R.J., Pajevic, S., Jones, D.K., 2002. Virtual in vivo interactive dissection of white matter fasciculi in the human brain. Neuroimage 17, 77-94.

Drevets, W.C., Savitz, J., 2008. The subgenual anterior cingulate cortex in mood disorders. CNS Spectr. 13, 663-681.

DSM-IV, American Psychiatric Association, 2000. Diagnostic and statistical manual of mental disorders, Fourth Edition. . Text Revision (DSMIV-TR).

Ebert, D., Ebmeier, K.P., 1996. The role of the cingulate gyrus in depression: from functional anatomy to neurochemistry. Biol. Psychiatry 39, 1044-1050.

Edwards-Lee, T., Miller, B.L., Benson, D.F., Cummings, J.L., Russell, G.L., Boone, K., Mena, I., 1997. The temporal variant of frontotemporal dementia. Brain 120, 1027-1040.

Fehr, E., Fischbacher, U., 2003. The nature of human altruism. Nature 425, 785-791.

Fitzgerald, D.A., Posse, S., Moore, G.J., Tancer, M.E., Nathan, P.J., Phan, K.L., 2004. Neural correlates of internally-generated disgust via autobiographical recall: a functional magnetic resonance imaging investigation. Neurosci. Lett. 370, 91-96.

Friston, K., 2010. The free-energy principle: a unified brain theory? Nat. Rev. Neurosci. $11,127-138$.

Friston, K.J., Buechel, C., Fink, G.R., Morris, J., Rolls, E., Dolan, R.J., 1997. Psychophysiological and modulatory interactions in neuroimaging. Neuroimage 6, 218-229.

Gitelman, D.R., Penny, W.D., Ashburner, J., Friston, K.J., 2003. Modeling regional and psychophysiologic interactions in fMRI: the importance of hemodynamic deconvolution. Neuroimage 19, 200-207.

Heekeren, H.R., Wartenburger, I., Schmidt, H., Prehn, K., Schwintowski, H.P., Villringer, A., 2005. Influence of bodily harm on neural correlates of semantic and moral decision-making. Neuroimage 24, 887-897.

Jefferies, E., Ralph, M.A.L., 2006. Semantic impairment in stroke aphasia versus semantic dementia: a case-series comparison. Brain 129, 2132-2147.

Kedia, G., Berthoz, S., Wessa, M., Hilton, D., Martinot, J.L., 2008. An agent harms a victim: a functional magnetic resonance imaging study on specific moral emotions. J. Cogn. Neurosci. 20, 1788-1798.

Kondo, H., Saleem, K.S., Price, J.L., 2003. Differential connections of the temporal pole with the orbital and medial prefrontal networks in macaque monkeys. J. Comp. Neurol. 465, 499-523.

Kriegeskorte, N., Formisano, E., Sorger, B., Goebel, R., 2007. Individual faces elicit distinct response patterns in human anterior temporal cortex. Proc. Natl. Acad. Sci. USA 104, 20600-20605.

Krueger, F., McCabe, K., Moll, J., Kriegeskorte, N., Zahn, R., Strenziok, M., Heinecke, A., Grafman, J., 2007. Neural correlates of trust. Proc. Natl. Acad. Sci. USA 104, 20084-20089.

Lamb, R.B., 1974. Adam smith's system: sympathy not self-interest. J. Hist. Ideas 35, 671-682.

Lambon Ralph, M.A., Patterson, K., 2008. Generalization and differentiation in semantic memory: insights from semantic dementia. Ann. NY Acad. Sci. 1124, 61-76.

Lambon Ralph, M.A., Pobric, G., Jefferies, E., 2009. Conceptual knowledge is underpinned by the temporal pole bilaterally: convergent evidence from rTMS. Cereb. Cortex 19, 832-838.

Lambon Ralph, M.A., Sage, K., Jones, R.W., Mayberry, E.J., 2010. Coherent concepts are computed in the anterior temporal lobes. Proc. Natl. Acad. Sci. USA 107, 2717-2722.

Liu, W., Miller, B.L., Kramer, J.H., Rankin, K., Wyss-Coray, C., Gearhart, R., Phengrasamy, L., Weiner, M., Rosen, H.J., 2004. Behavioral disorders in the frontal and temporal variants of frontotemporal dementia. Neurology 62, 742-748.

Logothetis, N.K., Wandell, B.A., 2004. Interpreting the BOLD signal. Annu. Rev. Physiol. $66,735-769$.

Maldjian, J.A., Laurienti, P.J., Kraft, R.A., Burdette, J.H., 2003. An automated method for neuroanatomic and cytoarchitectonic atlas-based interrogation of fMRI data sets. Neuroimage 19, 1233-1239.

Moll, J., Schulkin, J., 2009. Social attachment and aversion in human moral cognition. Neurosci. Biobehav Rev 33, 456-465.

Moll, J., Eslinger, P.J., de Oliveira-Souza, R., 2001. Frontopolar and anterior temporal cortex activation in a moral judgment task - Preliminary functional MRI results in normal subjects. Arq. Neuropsiquiatr. 59, 657-664.

Moll, J., de Oliveira-Souza, R., Bramati, I.E., Grafman, J., 2002. Functional networks in emotional moral and nonmoral social judgments. Neuroimage 16, 696-703.

Moll, J., Zahn, R., de Oliveira-Souza, R., Krueger, F., Grafman, J., 2005. The neural basis of human moral cognition. Nat. Rev. Neurosci. 6, 799-809.

Moll, J., Krueger, F., Zahn, R., Pardini, M., de Oliveira-Souza, R., Grafman, J., 2006. Human fronto-mesolimbic networks guide decisions about charitable donation. Proc. Natl. Acad. Sci. USA 103, 15623-15628.

Moll, J., de Oliveira-Souza, R., Garrido, G.J., Bramati, I.E., Caparelli-Daquer, E.M.A., Paiva, M.L.M.F., Zahn, R., Grafman, J., 2007. The self as a moral agent: linking the neural bases of social agency and moral sensitivity. Soc. Neurosci. 2, 336-352.

Moll, J., de Oliveira-Souza, R., Zahn, R., 2008. The neural basis of moral cognitionsentiments, concepts, and values. Year Cogn. Neurosci. 2008 (1124), 161-180. 
Neary, D., Snowden, J.S., Gustafson, L., Passant, U., Stuss, D., Black, S., Freedman, M., Kertesz, A., Robert, P.H., Albert, M., Boone, K., Miller, B.L., Cummings, J., Benson, D.F 1998. Frontotemporal lobar degeneration: a consensus on clinical diagnostic criteria. Neurology 51, 1546-1554.

O'Connor, L.E., Berry, J.W., Weiss, J., Gilbert, P., 2002. Guilt, fear, submission, and empathy in depression. J. Affect. Disord. 71, 19-27.

O'Doherty, J., Kringelbach, M.L., Rolls, E.T., Hornak, J., Andrews, C., 2001. Abstract reward and punishment representations in the human orbitofrontal cortex. Nat. Neurosci. 4, 95-102.

Patterson, K., Nestor, P.J., Rogers, T.T., 2007. Where do you know what you know? The representation of semantic knowledge in the human brain. Nat. Rev. Neurosci. 8 976-987.

Rankin, K.P., Gorno-Tempini, M.L., Allison, S.C., Stanley, C.M., Glenn, S., Weiner, M.W., Miller, B.L., 2006. Structural anatomy of empathy in neurodegenerative disease. Brain 129, 2945-2956.

Ressler, K.J., Mayberg, H.S., 2007. Targeting abnormal neural circuits in mood and anxiety disorders: from the laboratory to the clinic. Nat. Neurosci. 10, 1116-1124.

Rogers, T.T., Ralph, M.A.L., Garrard, P., Bozeat, S., McClelland, J.L., Hodges, J.R., Patterson, K., 2004. Structure and deterioration of semantic memory: a neuropsychological and computational investigation. Psychol. Rev. 111, 205-235.

Rogers, T.T., Hocking, J., Noppeney, U., Mechelli, A., Gorno-Tempini, M.L., Patterson, K., Price C.J., 2006. Anterior temporal cortex and semantic memory: reconciling findings from neuropsychology and functional imaging. Cogn. Affect. Behav. Neurosci. 6, 201-213.

Rogers, B.P., Morgan, V.L., Newton, A.T., Gore, J.C., 2007. Assessing functiona connectivity in the human brain by fMRI. Magn. Reson. Imaging 25, 1347-1357.

Rolls, E.T., 2004. The functions of the orbitofrontal cortex. Brain Cogn. 55, 11-29.

Ross, L.A., Olson, I.R., 2010. Social cognition and the anterior temporal lobes. Neuroimage 49, 3452-3462.

Schmahmann, J.D., Pandya, D.N., Wang, R., Dai, G., D'Arceuil, H.E., de Crespigny, A.J. Wedeen, V.J., 2007. Association fibre pathways of the brain: parallel observations from diffusion spectrum imaging and autoradiography. Brain 130, 630-653.

Seymour, B., Singer, T., Dolan, R., 2007. The neurobiology of punishment. Nat. Rev. Neurosci. 8, 300-311.

Shin, LM. Dougherty, D.D., Orr, S.P., Pitman, R.K. Lasko, M., Macklin, M.L, Alpert, N.M. Fischman, A.J., Rauch, S.L., 2000. Activation of anterior paralimbic structures during guilt-related script-driven imagery. Biol. Psychiatry 48, 43-50.
Simmons, W.K., Reddish, M., Bellgowan, P.S.F., Martin, A., 2009. The selectivity and functional connectivity of the anterior temporal lobes. Cereb. Cortex bhp149.

Small, D.M., Zatorre, R.J., Dagher, A., Evans, A.C., Jones-Gotman, M., 2001. Changes in brain activity related to eating chocolate - From pleasure to aversion. Brain 124 $1720-1733$.

Stephan, K.E., 2004. On the role of general system theory for functional neuroimaging. J. Anat. 205, 443-470.

Takahashi, H., Yahata, N., Koeda, M., Matsuda, T., Asai, K., Okuboc, Y., 2004. Brain activation associated with evaluative processes of guilt and embarrassment: an fMRI study. Neuroimage 23, 967-974.

Talairach, J., Tournoux, P., 1988. Co-planar stereotaxic atlas of the human brain. Thieme Medical Publishers, New York.

Tsukiura, T., Suzuki, C., Shigemune, Y., Mochizuki-Kawai, H., 2008. Differential contributions of the anterior temporal and medial temporal lobe to the retrieva of memory for person identity information. Hum. Brain Mapp. 29, 1343-1354.

Tzourio-Mazoyer, N., Landeau, B., Papathanassion, D., Crivello, F., Edard, O., Delcroix, N. Mazoyer, B., Joliof, M., 2002. Automated anatomical labelling of activations in spm using a macroscopic anatomical parcellation of the MNI MRI single-subject brain. Neuroimage 15, 273-289.

Vogt, B.A. Pandya, D.N., Rosene, D.L, 1987. Cingulate cortex of the rhesus-monkey. 1. Cytoarchitecture and thalamic afferents. J. Comp. Neurol. 262, 256-270.

Vogt, B.A., Nimchinsky, E.A., Vogt, L.J., Hof, P.R., 1995. Human cingulate cortex-surfacefeatures, flat maps, and cytoarchitecture. J. Comp. Neurol. 359, 490-506.

Weiss, J., Sampson, H., O'Connor, L., 1995. How psychotherapy works: the findings of the San Francisco Psychotherapy Research Group. Bull. Psychoanal. Res. Soc. 4.

Zahn, R., Moll, J., Krueger, F., Huey, E.D., Garrido, G., Grafman, J., 2007. Social concepts are represented in the superior anterior temporal cortex. Proc. Natl. Acad. Sci. 104 6430-6435.

Zahn, R., Moll, J., Paiva, M., Garrido, G., Kruger, F., Huey, E.D., Grafman, J., 2009a. The neural basis of human social values: evidence from fMRI. Cereb. Cortex 19, 276-283.

Zahn, R., Moll, J., Iyengar, V., Huey, E.D., Tierney, M., Krueger, F., Grafman, J., 2009b. Social conceptual impairments in frontotemporal lobar degeneration with right anterior temporal hypometabolism. Brain 132, 604-616.

Zahn, R. de Oliveira-Souza, R. Bramati, I. Garrido, G. Moll, J. 2009c. Subgenual cingulate activity reflects individual differences in empathic concern. Neurosci. Lett. $457,107-110$ 